\title{
A Study on CRM and Its Customer Segmentation Outsourcing Approach for Small and Medium Businesses
}

\author{
Feng Qian \\ Institute of Management Science \& Information Engineering, \\ Hangzhou Dianzi University, Hangzhou 310018, Zhejiang, P.R. China gianf@bdu.edu.cn
}

\begin{abstract}
Supported by technologies of Customer Satisfaction, Information Technology, and Data Mining, etc., CRM aims to enhance the effectiveness and performance of the businesses by improving the customer satisfaction and loyalty. CRM is now becoming a popular management methodology in manufacturing, sales, marketing, and finance. In China, there are a lot of small and medium businesses. For these businesses, sourcing CRM services on the web is a key business tactics for reducing the total ownership costs and implementation risks linked to "big bang" CRM implementations. In this paper, first, the architecture and contents of CRM approach for small and medium businesses were discussed according to the management characteristics. Second, contributes to the eCRM implementation landscape by providing a detailed account of the business process design and implementation support for a customer segmentation outsourcing.
\end{abstract}

Keywords: Customer relationship management, Customer segmentation, Outsource

\section{INTRODUCTION}

Customer Relationship Management (CRM) has become a leading business strategy in highly competitive business environment. CRM can be viewed as "managerial efforts to manage business interactions with customers by combining business processes and technologies that seek to understand a company's customers" [1]. Companies are becoming increasingly aware of the many potential benefits provided by CRM. Some potential benefits of CRM are as follows: (1) Increased customer retention and loyalty, (2) Higher customer profitability, (3) Creation value for the customer, (4) Customization of products and services, (5) Lower process, higher quality products and services [2].

When evaluating customer profitability, marketers are often reminded of the 80/20 rule $(80 \%$ of the profits are produced by top $20 \%$ of profitable customers and $80 \%$ of the costs are produced by top $20 \%$ of unprofitable customers) [3].

The core parts of CRM activities are understanding customers' profitability and retaining profitable customers. So, we describe CRM as an interactive approach that achieves an optimum balance between corporate investments and the satisfaction of customer needs in order to generate maximum profits. This entails: (1) acquiring and continuously updating data on customer needs, motivations, and behavior over the lifetime of the relationship. (2) continuously improving performance through a

Please use the following format when citing this chapter:

Qian, F., 2007, in IFIP International Federation for Information Processing, Volume 255, Research and Practical Issues of Enterprise Information Systems II Volume 2, eds. L. Xu, Tjoa A., Chaudhry S. (Boston: Springer), pp. 1387-1394. 
process of learning from successes and failures. (3) integrating marketing, sales, and service activities to achieve a common goal. (4) implementing appropriate systems to support customer knowledge acquisition, sharing, and the measurement of CRM effectiveness.

Despite the growing importance of CRM in revenue assurance and expansion, the spiraling total cost of ownership linked to CRM solutions and the high failure rates of CRM projects have prevented and/or delayed CRM uptake decisions by businesses, especially small and medium businesses in China. To avoid the potential high costs of mega failures and to develop CRM capabilities at an incremental pace, some organizations are opting for a piece-meal and flexible approach to CRM uptake. In this approach, business processes are mapped, prioritized, and CRM functionality is acquired/outsourced from vendors following a business case driven, incremental, and best of breed strategy [4]. The growing importance of outsourcing and piecemeal approaches to CRM uptake has been spotted by the major CRM market players and is also driving vendors to un-bundle CRM solutions into portfolios of reconciled with third party solutions. The web architecture and protocols are also playing a major role in the unbundling process and in the interface reconciliation process helping to provide the infrastructure for the integration and interoperation of different solutions. Moreover, the web is also becoming the preferred model for deploying front-end CRM e-services following an application services provider (ASP) delivery model.

\section{ARCHITECTURE OF CRM FOR SMALL AND MEDIUM BUSINESSES}

To integrate marketing, sales, and service activities, CRM requires the strong integration of business processes that involve customers. The architecture (figure 1) incorporates business processes in small and medium businesses that are regarded as relevant to $\mathrm{CRM}$ in most of the literature analyzed. We distinguish among three categories of CRM processes:

- CRM delivery processes: Processes with direct customer contact that are designed to cover part of the customer process (sales management, service management, complaint management).

- CRM support processes: Processes with direct customer contact that are not designed to cover part of the customer process, but to fulfill supporting functions within the CRM context (market research, loyalty management).

- CRM analysis processes: Processes that consolidate and analyze customer knowledge that has been collected in other CRM processes. The analysis results are passed on to the CRM delivery and support processes as well as to the service innovation and service production processes to improve their effectiveness (customer profiling, customer segmentation). 


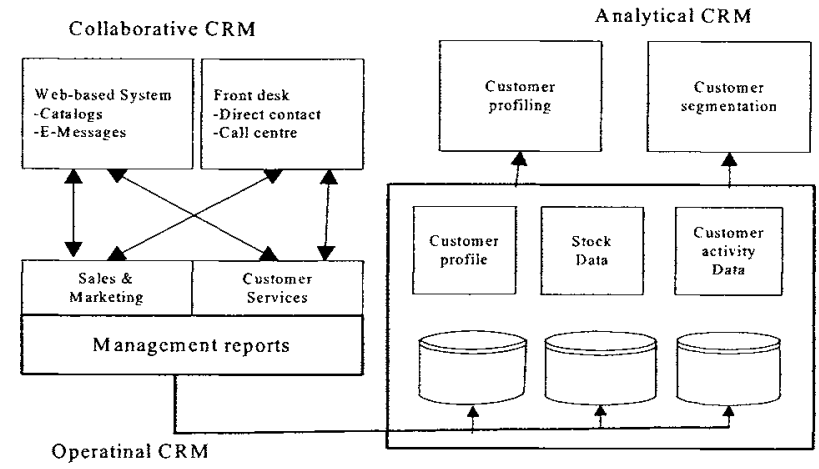

Figure 1. Architecture for CRM in Small and Medium Businesses

Next, we provide a short overview of each CRM process.

\subsection{CRM Delivery Processes}

The objectives of sales management are to understand the needs of a current or prospective known) customer as completely as possible, to consult the customer on possible alternatives with which to satisfy his needs, and finally to submit an offer, and close a deal. Therefore sales management covers the customer process phases of need articulation, evaluation, and buying.

Service and complaint management cover the customer process phases of using and expiry/renewal of a service contract complementarily. Service management is the planning, realization and control of measures for the provision of services. Examples include contract management and information services in the after-sales phase.

Customers' articulated dissatisfaction is received, processed, and communicated in the enterprise within the scope of complaint management. The objectives are to improve customer satisfaction in the short-run by directly addressing problems that led to complaints, and to support a continuous improvement process to avoid complaints in the long-run.

\subsection{CRM Support Processes}

Market research is the systematic design, collection, analysis, and reporting of data and findings relevant to a specific marketing situation facing the business.

Loyalty management is the planning, realization, control, and monitoring of measures to optimize the duration and intensity of relationships with customers. Exemplary measures are loyalty programs such as airlines' frequent flyer programs, but also churn management to identify customers who are in danger of migrating to competitors at an early stage. 


\subsection{CRM Analysis Processes}

Customer profiling is the analysis of current knowledge about customers in order to classify and characterize each customer, for example with regard to his value for the business, loyalty, and preferences regarding products and communication channels. Sales management, service management, complaint management, and loyalty management processes use the results of customer profiling to address customers in a more personalized way. Conversely, the objective of segmentation is the development of homogenous customer segments that have different product and service needs. Customer segments are the basis for the development of variants in a business's product and service portfolio.

Multi-channel management is a cross-functional activity and is responsible for the synchronization of the CRM delivery and support processes. It is the coordinated development, design and control of product and data flows to and from customers over different media and communication channels.

\section{ECRM AND CUSTOMER SEGMENTATION OUTSOURCING APPROACH}

Several dimensions of CRM functionality can be provided to businesses as services delivered by ASPs, building on the web infrastructure. An example of some possible services outsourcing is customer profiling and customer segmentation.

\subsection{Web-based CRM}

In today's competitive business environment, the ability to effectively and efficiently manage the flow of information is a vital competency. Businesses must be able to integrate their internal business processes horizontally and vertically, and they are increasingly required to support federated business processes and integrate application silos in existing information systems [5, 6]. Web Services [7], as an emerging form of the service-oriented architecture for distributed computing, have the potential to simplify the integration task and allow businesses to "stitch" together many different application components.

There are two important reasons driving businesses to use web services:

- Web services allow independent businesses with heterogeneous IT platforms to connect and collaborate with each other across the Internet. New business partnerships can be constructed dynamically and automatically, since web services ensure complete interoperability between systems.

- Web services allow disparate systems within an enterprise, e.g. financial systems and CRM systems, to communicate with ease. Web services also make the integration of legacy information systems into new generation information systems become feasible. Legacy systems can be wrapped in a web service facade without changing the way that customers access the service. The process of creating an integrated infrastructure for linking disparate systems, 
A Study on CRM and Its Customer Segmentation Outsourcing Approach for Small and

applications, and data sources across the corporate enterprise is known as Enterprise Application Integration (EAI).

\subsection{Customer Segmentation}

A framework for customer segmentation based on customer value is organized into two phases. Phase 1 explains the preparation steps to be conducted before defining the customer value. In phase 2 evaluate the customer value from some viewpoints --current value, potential value or customer loyalty. After segmenting the customer base with three viewpoints, a segment analysis is performed according to the segmentation results.

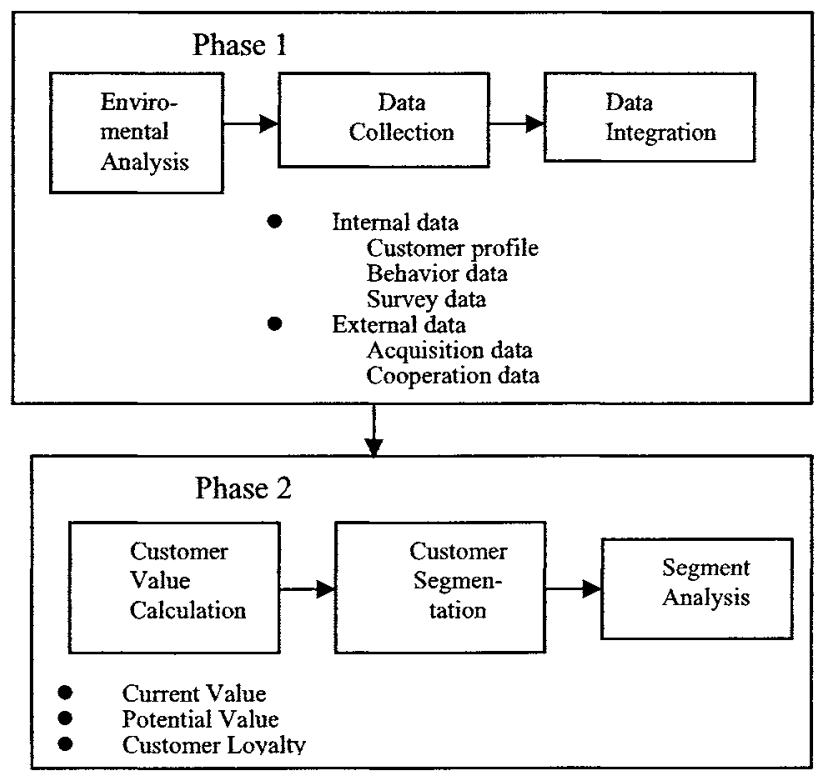

Figure 2. Framework for Customer Segmentation

\subsection{Customer Segmentation Business Process Design}

Figure 3 illustrates the details of the customer segmentation business process performed by the ASP. The designed business process is a web-based process in which a website acts as the service front-end for the ASP delivering the specific CRM functionality -- customer segmentation. The client will be able to use the customized segmentation application on-demand through the website to classify his/her new acquired customer data. The segmentation results of the new acquired customers can 
be displayed instantly. The customized web service can also be invoked from the CRM system running in the client's business. The functionality of the client's CRM system, hence, is extended by the web service.

The sequence of the designed business process to provide a requested customer segmentation application can be described as follows: The process begins with the request of a business client for a customized customer segmentation application to classify his/her customer data. To request a desired segmentation application, the client needs to submit his/her segmentation requirements and a XML file containing his/her sample customer data through the ASP's segmentation services website.

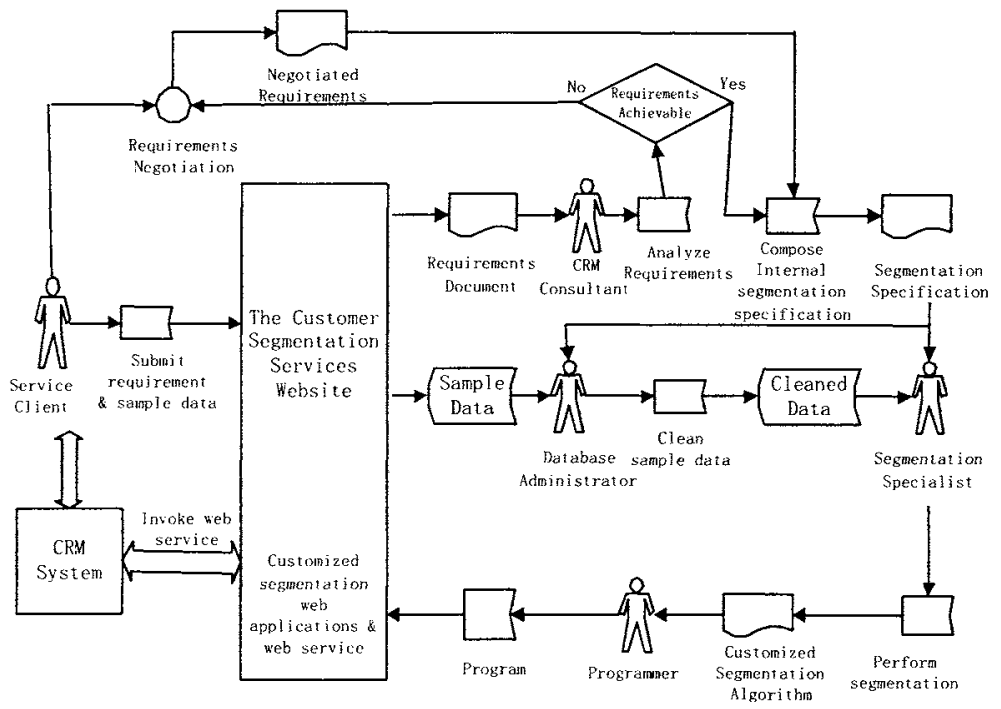

Figure 3. Customer Segmentation Business Process

In the segmentation requirements, the service client may state out how he/she wants to segment his/her customers, which segmentation technique he/she wants to employ to classify his/her customer data, how accurate the segmentation results should be, and so on.

Regarding the sample customer dataset, it is used for performing data classification, so that appropriate segmentation algorithms can be identified to construct the requested segmentation application based on the client's segmentation requirements. When the client submits the sample dataset, he/she also needs to provide a document which contains the description of the attributes in the sample dataset.

Once the client submits his/her segmentation requirements to the system, the system will create a document recording the client's segmentation requirements. CRM consultants in the back-office of the ASP will analyze the client's requirements. The CRM consultants are experts familiar with CRM and customer segmentation. 
If CRM consultants consider the client's segmentation requirements are unreasonable or unachievable (e.g. the client requires a data mining algorithm-based segmentation application that can classify his new customer data with $100 \%$ accuracy.), they will contact the client to negotiate the requirements, so that they can achieve an agreement on the revised segmentation requirements.

When the segmentation requirements are considered to be achievable, CRM consultants will compose an internal segmentation specification and send it to database administrators and segmentation specialists. Segmentation specialists are the staff specialized in data mining and statistical methods for customer segmentation. Segmentation specialists will choose appropriate methods and software to perform data classification according to the internal segmentation specification.

Before segmentation specialists use the client's sample customer data to perform data classification, the sample customer data submitted through the segmentation services website by the client may need to be cleaned if the data has poor quality (e.g. some records have missing values in certain fields such as address or date of birth). The data cleaning action is performed by database administrators after database administrators receive the internal segmentation specification.

After segmentation specialists identify the most appropriate classifier model to classify the client's sample customer data according to the segmentation requirements, they document the segmentation algorithm of the classifier model and send it to programmers. Based on the segmentation algorithm, programmers program the customized web service and web application which will be integrated into the customer segmentation services website. The customized web service and web application can only classify the customer data in which the attributes must be the same as the ones in the sample data submitted by the client before.

Once the requested customized segmentation web application and web service are integrated into the customer segmentation services website, the service client will be informed that his/her requested service is available. The client will be able to use the customized segmentation application to classify his/her new acquired customer data in the website. Moreover, the client can also invoke the customized segmentation web service in his/her CRM system to classify his/her customer data.

Based on the above description of the designed business process, it is known that the supporting customer segmentation services website is critical to implement the designed business process. The customer segmentation services website is not only the place where service clients request customized segmentation applications, but also the place where service clients consume their segmentation applications.

\section{CONCLUSIONS}

Building on previous research and comprehensive analysis of research literature, we developed a CRM reference architecture. This architecture can be used for the description and classification of CRM approaches in small and medium businesses. And then, take customer segmentation as an example, we introduced an outsourcing approach to CRM and a detailed web-based customer segmentation business process provided by ASPs was designed. 
The development of CRM capabilities by incrementally sourcing and combining "best of breed" services from different providers is a key lever for reducing implementation risks and costs linked to CRM projects. For small and medium businesses in China, central to a "best of breed" and incremental CRM implementation strategy is the unbundling of CRM functionality into a portfolio of services that can be sourced from application service providers and/or developed internally within the client organization.

\section{REFERENCES}

1. J. Kim, E. Suh, and H. Hwang, A model for evaluating the effectiveness of CRM using the balanced scorecard. Journal of Interactive Marketing. Volume 17, Number 2, pp.5-19, (2003).

2. D.J. Craig and P. Bodorik, Enabling and measuring electronic customer relationship management readiness, in Proc. of the 34th anmual Hawaii international conference on system sciences organizational systems and technologies track (2001), pp.1-10.

3. R.S. Duboff, Marketing to maximize profitability. The Joumal of Business Strategy. Volume 13, Number 6, pp.10-13. (1992).

4. D. Hancock, H. Galal, and F.P. Sampaio, CRM in a shoestring: Achieving performance impact through customer relationship management, Knowledge Object (McKinsey \& Company Inc., 2002)

5. W.A. Estrem, An Evaluation Framework for Deploying Web Services in the Next Generation Manufacturing Enterprise, Robotics and Computer-Integrated Manufacturing. Volume 19, Number 6, pp.509-519, (2003).

6. P.B. Seybold, The Five Waves of Customer Relationship Management ---How CRM Functionality and Architecture Have Evolved, Business Briefing: Data Management \& Storage Technology. pp.1-2, (2002).

7. F. Buttle, Customer Relationship Management Concepts and Tools (Elsevier publishers, 2004). 